\title{
Un estudio descriptivo de Personalidad, salud y estrés en estudiantes de Secundaria (16-19 años)
}

Eva Naper Jensen*
Sven Svebak ${ }^{\star *}$
K Gönnar Götestam**

* The County Municipality of Sör-Tröndelag, School Division, Trondheim

** Norwegian University of Science and Technology, The Medical Faculty

NORWAY

RESUMEN - Se distribuyó un cuestionario a 1.162 estudiantes de secundaria, de los cuales a $1.069(92 \%)$ se les administraron los siguientes instrumentos: Life Regard Index (Índice de Perspectiva Vital), Positive and Negative Mood (Estado de ánimo Positivo y Negativo, Zung Depression Self-Rating Scale (escala autoadministrada de depresión de Zung), escala de neuroticismo del EPQ (Cuestionario de Personalidad de Eysenck), Sense of Humor Questionnaire (SHQ-6, Cuestionario sobre el Sentido de Humor), Tension and Effort Stress Inventory (Cuestionario de Tensión y Estrés de esfuerzo), y Ursin Health Inventory (Cuestionario de Salud Ursin) para las quejas somáticas. Los resultados mostraron diferencias tanto por sexo como por zona rural/urbana; es decir, los chicos y los estudiantes de zonas rurales obtuvieron las puntuaciones positivas más altas. Alrededor del 15\% mostró depresión importante, y un 30\% adicional tenía síntomas depresivos. Las puntuaciones altas en sentido del humor y en el índice de consideración vital se correlacionaron con puntuaciones bajas en depresión.

\section{Introducción}

La reforma propuesta por el gobierno noruego ("School Reform 94") impulsó un cambio en las condiciones de trabajo y de aprendizaje en todo el sistema educativo en Noruega. En conexión con la implantación de esta reforma, el condado de Sör-Trönde- lag inició en 1996 un proyecto de promoción de la salud en educación secundaria con el título: "Un colegio con sentido". El objetivo de este proyecto fue desarrollar nuevos procedimientos de trabajo, tanto para los colegios como para los servicios sanitarios, que son los que mantienen y promocionan la salud y el aprendizaje de los estudiantes jóvenes en su vida diaria. El proyecto fue parte de 
las actividades desarrolladas por el Ministerio de Salud y Asuntos Sociales noruego, y se llevó a cabo en colaboración con la Universidad Noruega de Ciencia y Tecnología, Facultad de Medicina. La reforma, que dio lugar a muchos cambios, estaba aprobada por el gobierno noruego. Estos cambios tuvieron consecuencias para el papel tradicional del profesor, así como para el de los estudiantes. El rol del profesor se concebía como un rol más parecido al de un entrenador de gente joven en desarrollo. Los estudiantes jóvenes tenían que asumir más responsabilidad sobre su propio aprendizaje y tenían que contemplar la posibilidad de participar en el planteamiento y evaluación de su aprendizaje (Björgen 1993). La condición para ello es que debe haber una cooperación mejor y segura entre el profesor y los estudiantes y entre los propios estudiantes en su aprendizaje diario. Los estudiantes quieren un cambio, y subrayan la falta de comunicación entre el profesor y el estudiante, el "lazo ausente". La pregunta es si realmente queremos una nueva forma de trabajo en nuestros colegios (Lindsley 1992). Aunque hayamos adquirido conocimientos y haya métodos educativos efectivos disponibles, los cambios en la forma de trabajo en el colegio van muy despacio.

El colegio es el lugar de trabajo de los estudiantes. El ambiente del colegio es importante, ya que constituye el lugar de trabajo de la gente joven en un período de sus vidas que es esencial para su desarrollo como personas (desarrollo humano), su desarrollo de conceptos y el crecimiento de sus capacidades intelectuales. Como el desarrollo de su eficacia intelectual es, en gran medida, un constructo social basado en evaluaciones de sus rendimientos en diferentes asignaturas académicas, en comparaciones sociales repetidas con los éxitos de sus iguales y en respuestas de sus profesores (Bandura 1997), nuestro objetivo debe ser el desarrollo de un colegio con un buen ambiente de aprendizaje y abrir comunidades donde tanto adultos como adolescentes sean tratados con cuidado $\mathrm{y}$ respeto.

Muchos de los problemas que presenta la gente joven son de tipo psicosocial o psicosomático. La "School Reform 94" hace del trabajar para el desarrollo de la personalidad del estudiante una obligación para la plantilla del colegio. Es importante que los estudiantes desarrollen un entendimiento de sí mismos y de su potencial de aprendizaje en cooperación con sus compañeros estudiantes, profesores y otros profesionales. La parte general del programa dice "el progreso depende no sólo de cómo los profesores funcionan en relación a cada estudiante, sino también de cómo hacen que los estudiantes funcionen en relación a cada uno de los otros. En un buen equipo, los miembros del mismo realzan la calidad del trabajo de cada uno de los otros" (Core Curriculum for Primary, Secondary and Adult Education in Norway 1997).

La reforma estimula el desarrollo de la "persona" y de los valores más humanísticos, paralelamente al desarrollo del aprendizaje y de las habilidades académicas. Esto requiere unos roles modificados del estudiante y del profesor, conceptos modificados del aprendizaje y una comunicación inteligente y buena cooperación entre la gente, como base para la salud y el aprendizaje. El objetivo es hacer capaces a los estudiantes de enfrentarse a los retos y cargas de la vida diaria.

Es esencial enfatizar el hecho de que el trabajo realizado, en el proyecto mencionado, está dirigido a todos los estudiantes, no particularmente a los estudiantes que tienen problemas identificables. El proyecto incluye a todos los que forman la sociedad del colegio. Ésta ha sido un área olvidada en la educación secundaria. ¿Necesitamos cambiar la educación de los profesores? 
De entre las actividades que se han desarrollado podemos mencionar "comienzo activo del año escolar" (los estudiantes participan en el proceso de aprendizaje y planificación del año escolar), "grupos centrales" (familiarización, promover la colaboración), "crear actitudes positivas" (hacer comentarios positivos), "adaptación y evaluación" (participación en el proceso de evaluación) y "métodos de trabajo modificados para los servicios sanitarios" (aproximación a la prevención primaria de salud).

Elegimos actividades que se centraron en la relación entre los diversos agentes del colegio. La razón de por qué elegimos este foco de atención es que creemos que las relaciones son el fundamento de cualquier sociedad (nosotros solemos decir que nuestra existencia se basa en las relaciones). Por tanto, para los participantes en este proyecto ha sido esencial intentar responder a la pregunta: ¿Cómo podemos organizar juntos el trabajo para ser capaces de crear juntos?

Uno de los objetivos principales del proyecto fue adquirir experiencia para integrar el trabajo de promoción de la salud y preventivo en las actividades escolares ordinarias. También quisimos ilustrar la importancia de la participación del estudiante en sus condiciones de aprendizaje. Una de nuestras experiencias más importantes fue que hay razones para hacer algunas preguntas críticas respecto a la forma en que trabajan los colegios hoy en día.

\section{$¿$ Experimentan los estudiantes, hoy en día, el colegio como algo con sentido?}

La experiencia y los análisis de investigaciones científicas indican que deben hacerse cambios. Ya que el colegio es el lugar de trabajo para los estudiantes, es responsable tam- bién de los ambientes de aprendizaje. Es muy importante que los estudiantes experimenten una vida diaria escolar con más sentido que hasta ahora. Entre otras cosas, estos pasan más tiempo que nunca en el colegio y menos en sus casas (Leenars y Wenckstern 1991).

\section{¿Es realista un colegio de promoción de la salud para prevenir problemas?}

Mediante la entrevista a diferentes grupos de estudiantes se describen sus opiniones sobre el ambiente escolar y el potencial de cambio. Las entrevistas indican que el entorno de trabajo se caracteriza por unas expectativas altas (por parte de los estudiantes mismos, padres y profesores), así como por obtener buenos resultados (notas) e ir bien (progresar) en el colegio. Hay muchas dificultades en el trabajo diario en el colegio, y los profesores son responsables de las cuestiones relacionadas con su asignatura (esta dificultad es difícil de cambiar). El colegio trata básicamente sobre el logro individual y no sobre los valores colectivos, atención y solidaridad. Aquéllos que no reúnen los requisitos se eliminan y cada vez se remiten más estudiantes al psicólogo escolar. El colegio ha sido reducido a la introducción hacia nuestra sociedad moderna, eficiente y competitiva, mientras que deberíamos fomentar nuevos individuos mejores, no sólo copiar malas características de los adultos anteriores.

\section{¿Cuáles son las consecuencias prácticas de las nuevas guías, y cómo podríamos implantarlas?}

Hay tantas expectativas diferentes respecto a "la vieja idea de que lo importante son buenas notas en las diferentes asignaturas" que 
para los directores de los colegios es difícil, hoy en día, tomarse en serio las nuevas visiones escolares.

Podría ayudar quizás a nuestros directores de colegios hacer una pregunta como ésta: ¿Cuáles van a ser las consecuencias prácticas de nuestras nuevas reformas (nuevas formas de trabajo, nuevas visiones sobre el aprendizaje, nuevos servicios sanitarios y participación de los estudiantes) para nuestro colegio y cómo vamos a implantarlas?

Los máximos dirigentes escolares deben ser claros en sus prioridades y decidir si quieren que el colegio sea un lugar donde se enfatizan las relaciones sociales.

El rol del profesor tiene que ser más como el de un entrenador, motivando para que tenga lugar el aprendizaje. Se ha llevado a cabo un estudio preliminar para estudiar el efecto de mejorar las relaciones humanas en el trabajo académico y la situación laboral en el colegio (Jensen y Götestam 1998). Los estudiantes deberían tener la oportunidad de ser participantes activos en el proceso de aprendizaje antes que ser "una jarra que llenar". El foco de atención debería estar en las experiencias del estudiante que va al colegio, el estudiante como una persona, que se va a desarrollar en colaboración con los demás estudiantes y con los diferentes entrenadores.

Es necesario preguntar ¿qué tipo de aprendizaje es esencial tener con la intención de que cada uno dirija su vida en la sociedad de hoy? ¿Es posible y deseable, para que el trabajo se lleve a cabo, administrar el colegio y el trabajo del colegio de forma más colaborativa, de manera que los estudiantes se sientan más iguales, o sean aceptados como colaboradores, y el profesor sea consciente de su rol como líder?
Este líder tiene que hacer uso de una teoría de líder y unos pensamientos humanísticos nuevos. ¿Están preparados para esto los profesores?

Al final del proyecto, un grupo seleccionado de estudiantes, profesores, cuidadoras escolares y personas de la dirección del colegio participaron en un taller donde se presentaron y practicaron estas ideas. Este fue un gran reto para el sistema escolar (y sus actores), que no se ha cambiado apenas desde su creación.

El estudio al que se hace referencia en este proyecto pretendía recoger información sobre la vida diaria en el colegio, la salud y las condiciones de aprendizaje positivas y negativas y problemas. Esta información podría servir de base para los cambios, que son necesarios.

Quisimos evaluar el significado en la vida, predisposición a la ansiedad y al nerviosismo, grado de depresión, sentido del humor así como estados de ánimo placenteros y estados de desánimo, y las quejas somáticas.

\section{Método}

\section{Sujetos}

Se repartieron formularios de preguntas, descritos más abajo, a una muestra de 1.162 estudiantes, de los que 1.069 (tasa de respuesta: 92\%) eran de distintos institutos de Sör-Tröndelag. El cuestionario constaba de 8 páginas, y lo respondieron estudiantes de tres cursos distintos, y diferentes colegios, en la ciudad de Trondheim $(n=698)$ y en algunos de sus distritos $(\mathrm{n}=372)$, y de ambos sexos (524 chicas y 545 chicos). 


\section{Instrumentos}

Vida con sentido

El Life Regard Index (LRI; Índice de Perspectiva Vital; Battista y Almond 1973) se basa en un cuestionario de 28 ítems con dos subescalas (Metas en la vida; Consecución de las metas). Cada subescala tiene 14 ítems, la mitad formulada positivamente y la mitad negativamente. Se pide aquí a los sujetos que indiquen su propia opinión en una escala de 2 puntos ( 1 = estoy en desacuerdo, 2 = estoy de acuerdo).

\section{Depresión}

La escala autoadministrada de depresión de Zung (Zung Self-rating Depression Scale; SDS; Zung et al. 1965) contiene 20 ítems, puntuados en una escala de 4 puntos (la mitad de 1 a 4 y la otra mitad de 4 a 1), en la que las puntuaciones altas indican depresión.

\section{Eysenck Personality Questionnaire}

Se utilizaron los 12 ítems de Neuroticismo de la escala EPQ (Cuestionario de Personalidad de Eysenck; Eysenck, Eysenck y Barrett 1985) $(0=$ No, $1=$ Sí).

\section{Humor}

El Cuestionario sobre el Sentido del Humor (Sense of Humor Questionnaire, SHQ; Svebak 1996) tiene 21 ítems y tres subescalas (sensibilidad "meta-mensaje", humor que gusta "liking humor", expresión del humor "expressing humor"), puntuado en una escala de 4 puntos. En el presente estudio hemos empleado el cuestionario reducido, con sólo 6 ítems (SHQ-6).

Tensión y afrontamiento; estado de ánimo

El Tension and Effort Stress Inventory (TESI; Cuestionario de Tensión y Estrés de esfuerzo; Sveback 1990) tiene tres secciones: una centrada en el grado de estrés (presión, reto, demanda) al que uno se siente expuesto en el día a día (trabajo, familia, situación económica, el propio cuerpo). Otra sección trata sobre el grado de esfuerzo que uno hace para afrontar el estrés en las mismas áreas. La sección tiene cinco ítems, en los que las puntuaciones altas indican nivel alto de estrés o esfuerzo. Una tercera sección está compuesta de 8 ítems sobre los estados de ánimo negativos. Todos los ítems de TESI se puntúan en una escala de 7 puntos $(1=$ Nada; 7 = Mucho $)$.

\section{Quejas somáticas}

El Ursin Health Inventory (UHI; Cuestionario de Salud de Ursin; Ursin et al. 1988) incluye 27 ítems sobre los síntomas comunes de salud-enfermedad, o quejas somáticas. Se puntúan tanto la intensidad $(0=$ No dolor, 3 = Dolor grave) como la duración (030 días el mes pasado).

\section{Resultados}

\section{Vida con sentido}

La Figura 1 presenta los resultados del Life Regard Index (puntuaciones compuestas de sus dos subescalas, metas en la vida y Consecución de metas. Hubo una diferencia clara entre los entrevistados en distritos rurales y urbanos: los jóvenes de las zonas urbanas tenían un grado más alto de vida con sentido, en función de ambas variables. Hubo también diferencia entre los sexos, puntuando más alto los hombres en ambas subescalas.

\section{Estado de ánimo}

Generalmente, entre los entrevistados hay un grado más alto de estado de ánimo placentero, comparado con el estado de desánimo. Sólo la variable del estado de desánimo mostró alguna diferencia; las chicas refirieron más estados de ánimo negativos "durante los 
Vida con sentido

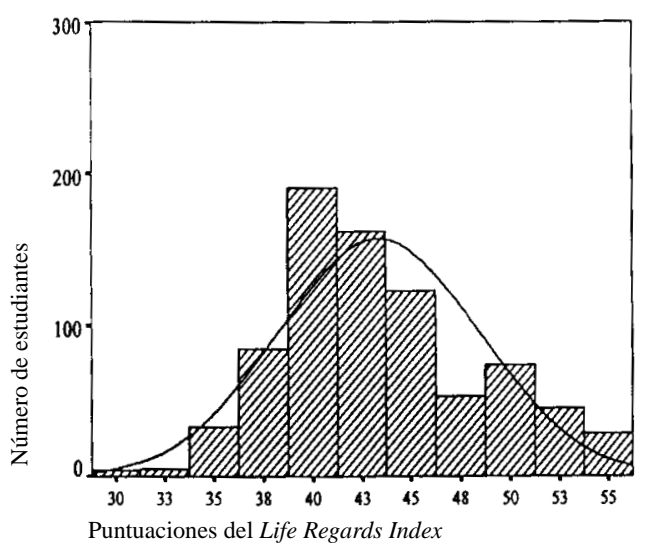

Vida con sentido

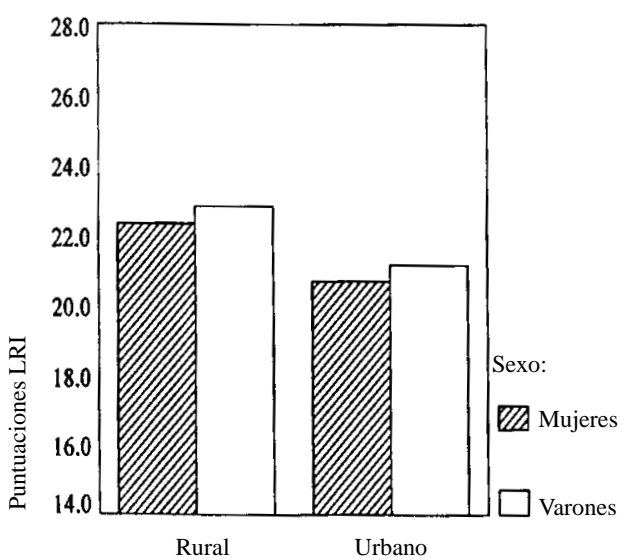

Figura 1. Puntuaciones compuestas del Life Regard Index sobre vida con sentido. Figura izquierda: distribución de frecuencias. Figura derecha: Distribución de puntuaciones por sexo y medio rural/urbano.

últimos treinta días" que los chicos. Los componentes más fuertes fueron furia, malhumor y culpa.

\section{Depresión}

Con la escala Zung, la mayoría de sujetos se encontraban dentro del área de normalidad (no-depresión), mientras que aproximadamente para un $35 \%$ podría describirse una depresión ligera o una reac-

Estados de ánimo placenteros

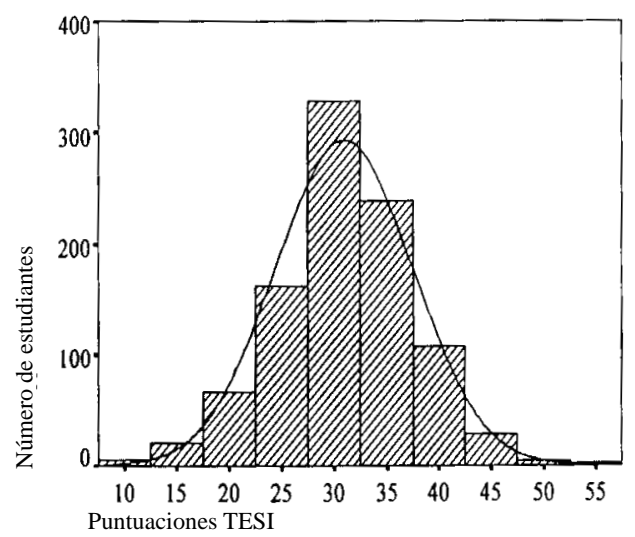

ción depresiva, y cerca de un $15 \%$ tenía depresión importante. Se halló más depresión en los sujetos de zonas rurales que en los de zonas urbanas, y las chicas estaban más deprimidas que los chicos.

\section{Eysenck Personality Questionnaire}

En la escala de Neuroticismo del EPQ, la mayoría de estudiantes obtuvieron puntuaciones medias-altas, y algunos muy altas o

\section{Estados de ánimo placenteros}

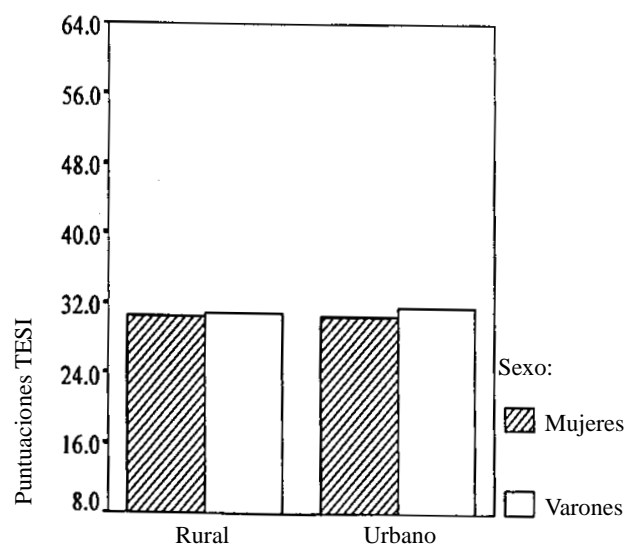

Figura 2. Estado de ánimo placentero. Figura izquierda: distribución de frecuencias. Figura derecha: Distribución de puntuaciones por sexo y medio rural/urbano. 
muy bajas de estado de ánimo disfórico. Los sujetos de zonas urbanas puntuaron más alto que los de zonas rurales, y las chicas puntuaron más alto que los chicos.

\section{Humor}

Con el Sense of Humor Questionnaire (SHQ-6), los sujetos de zonas rurales obtuvieron puntuaciones más altas en humor

Estados de ánimo desagradables

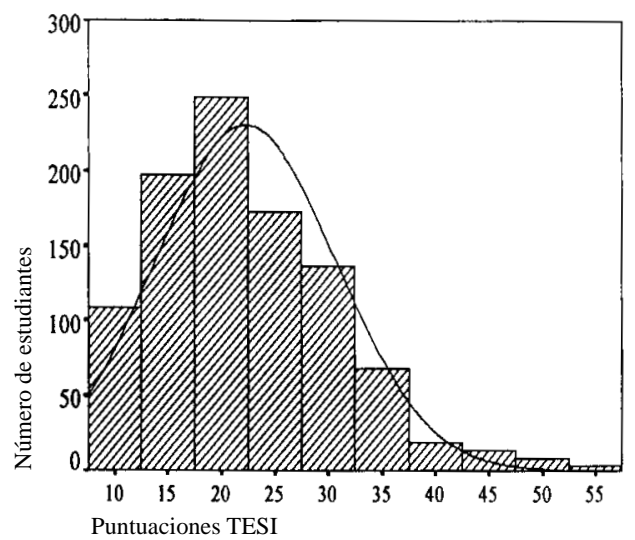

que los de zonas urbanas, mientras que no se encontraron diferencias por sexo.

\section{Estrés y esfuerzos relacionados}

Las puntuaciones en el Tensión and Effort Stress Inventory, no se diferenciaron entre urbano/rural para ninguno de los dos grupos de variables. En ambos, grado de tensión y grado de esfuerzo, las chicas mostraron puntuaciones más altas.

Estados de ánimo desagradables

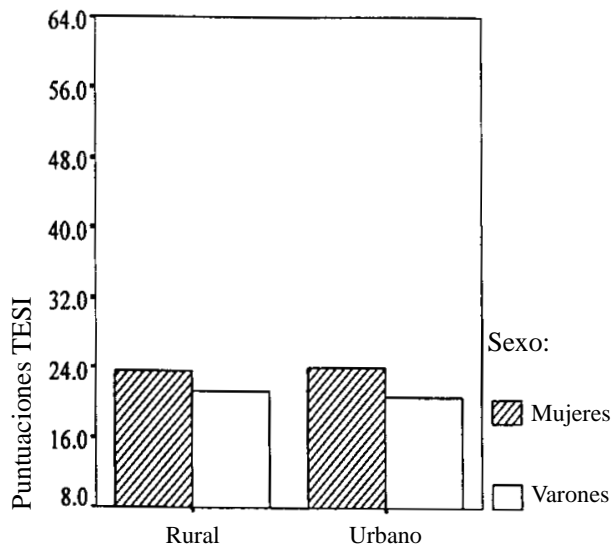

Figura 3. Estado de ánimo desagradables. Figura izquierda: distribución de frecuencias. Figura derecha: Distribución de puntuaciones por sexo y medio rural/urbano.

Depresión

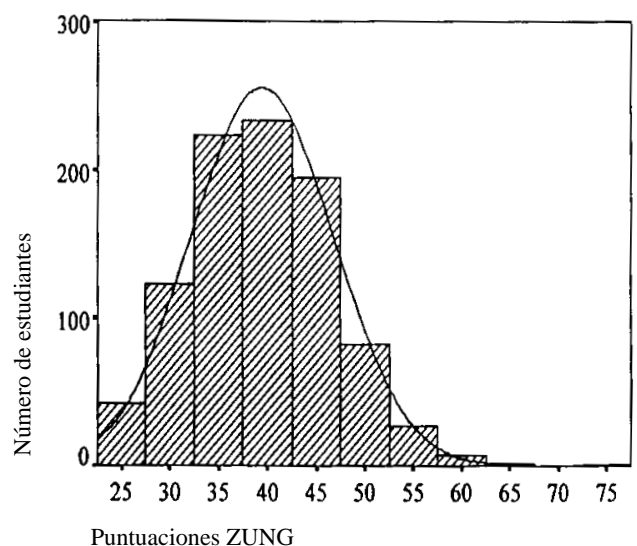

Depresión

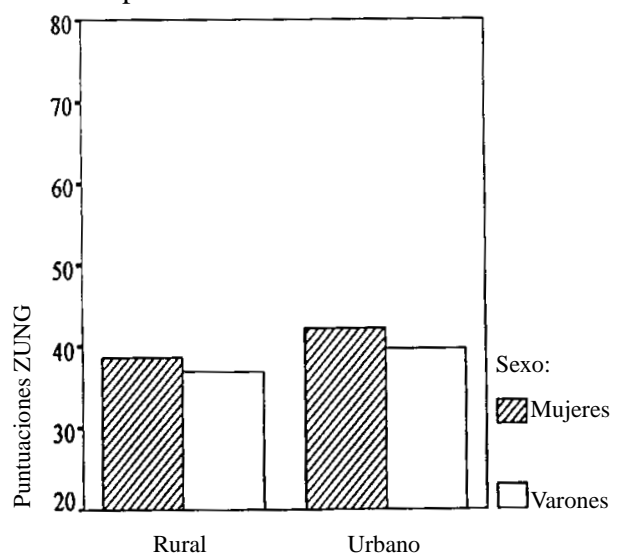

Figura 4. Grado de depresión, con criterios de la escala de Zung. Figura izquierda: distribución de frecuencias. Figura derecha: Distribución de puntuaciones por sexo y medio rural/urbano. 


\section{Quejas somáticas}

En el Ursin Health Inventory (UHI) la mayoría de estudiantes refirieron pocas quejas o ninguna, mientras que pocos de ellos refirieron un grado sustancial de quejas. Los tipos más prevalentes de quejas fueron dolor en brazos y piernas, dolor en el cuello, hombros y espalda, dolor de cabeza y problemas de sueño.

\section{Correlaciones}

Se computaron las correlaciones entre todas las variables principales. Muchas varia-

Tendencias disfóricas

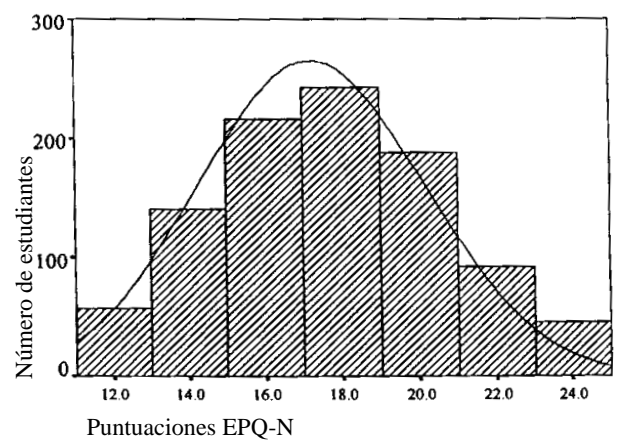

bles alcanzaron significación en esta muestra amplia pero, de acuerdo al principio de Bonferroni, sólo consideraremos las correlaciones por encima de 0,40 ( $p<0,0000)$. Se hallaron dos correlaciones esperadas, una entre el alcance de metas y metas en la vida. $(0,67)$ (dos partes del mismo cuestionario), y otra entre grado de tensión y grado de esfuerzo $(0,71)$ (dos partes del mismo cuestionario). Se hallaron, además, interesantes correlaciones entre depresión por una parte y sentido del humor $(-0,42)$ y vida con sentido $(-0,43)$, por la otra.

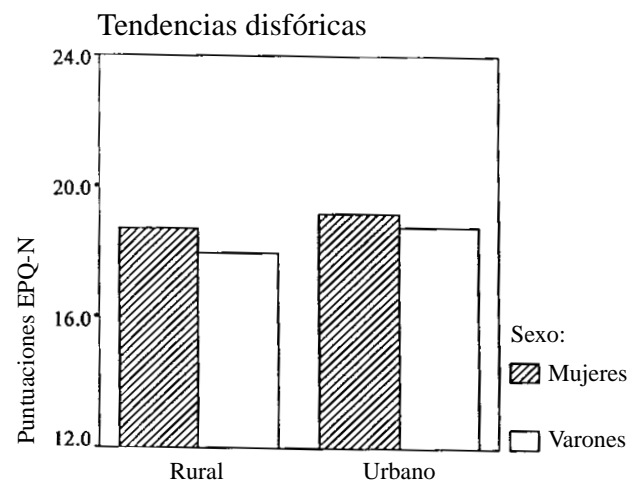

Figura 5. Neuroticismo EPQ. Figura izquierda: distribución de frecuencias. Figura derecha: Distribución de puntuaciones por sexo y medio rural/urbano.

Sentido del humor

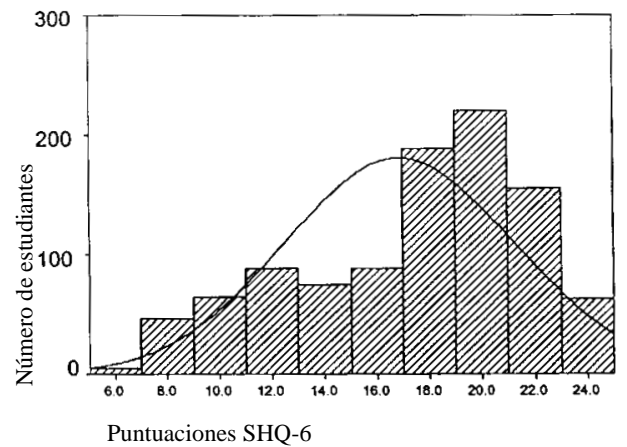

Sentido del humor

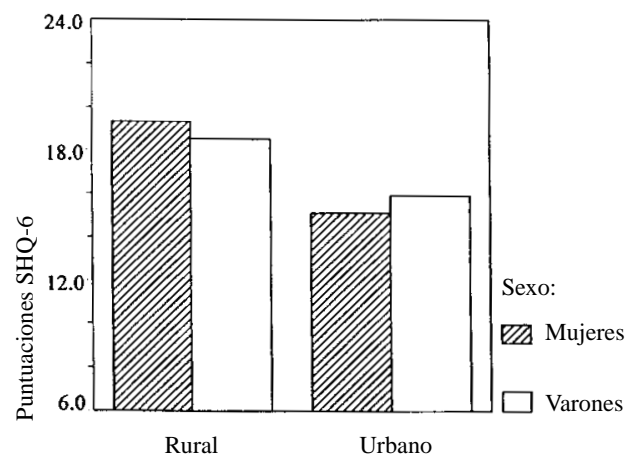

Figura 6. Sentido del humor (SHQ-6). Figura izquierda: distribución de frecuencias. Figura derecha: Distribución de puntuaciones por sexo y medio rural/urbano. 
Quejas somáticas

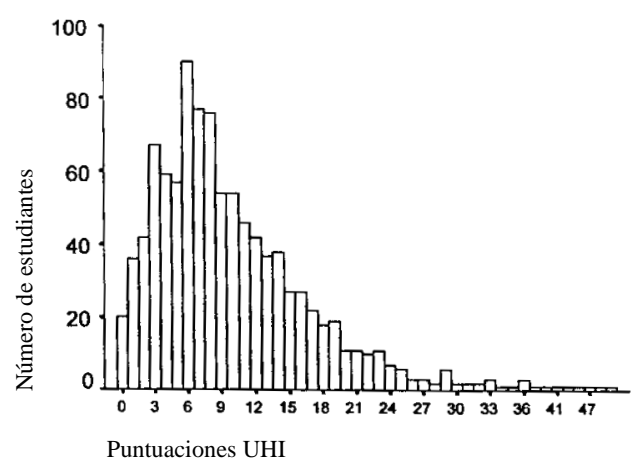

Quejas somáticas

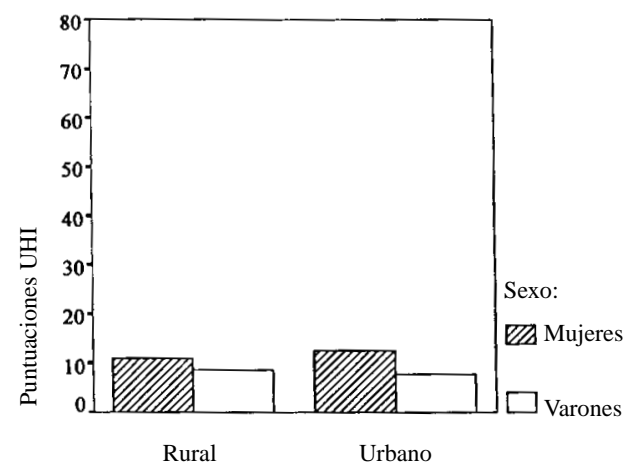

Figura 7. Quejas somáticas (puntuaciones totales). Figura izquierda: distribución de frecuencias. Figura derecha: Distribución de puntuaciones por sexo y medio rural/urbano.

\section{Discusión}

En el presente estudio, de acuerdo con la escala autoadministrada de depresión de Zung, un $15 \%$ de los estudiantes tenían una seria depresión, mientras que un $30 \%$ adicional tenía síntomas depresivos. Ésta es una frecuencia bastante alta de problemas depresivos en esta población adolescente. Una mirada más cercana a las variables de control que pueden contribuir a la explicación de la depresión: sentido del humor, alcance/consecución de metas, metas en la vida, estado de ánimo negativo, estado de ánimo positivo. La variable neuroticismo, sin embargo, no alcanzó significación, y por tanto, podría no explicar depresión.

A partir de estos resultados parece adecuado centrarse en la pregunta de si los adolescentes tienen una vida con sentido en el colegio. ¿O es una vida "puramente académica"? ¿Son las condiciones de trabajo para los estudiantes en el instituto consistentes con la "School Reform 94"? La reforma requiere nuevos roles para el estudiante y para el profesor. La educación del profesor, para los profesores que traba- jan en el instituto, es un tema de discusión. Los profesores de primaria parecen tener un mejor entendimiento de los seres humanos y de sus necesidades. ¿Tenemos que combinar la educación de los profesores de primaria y los profesores de instituto? No sólo necesitamos educación en los diferentes estudiantes, sino también el conocimiento que está disponible respecto a los adolescentes en diferentes situaciones como, por ejemplo, en el aprendizaje. ¿Tiene el profesor de instituto un interés real en los estudiantes jóvenes como seres humanos o es únicamente lo relativo a la asignatura lo que cuenta? El objetivo es hacer capaces a los estudiantes para enfrentarse a los retos y actividades de la vida cotidiana y que disfruten mientras aprenden, aunque el aprendizaje sea un trabajo duro.

La situación descrita es un reto inmenso para los colegios de hoy, y también tiene un potencial enorme para las mejoras, ambos en el bienestar de los estudiantes de los colegios, pero también probablemente una mejora en su rendimiento académico. 


\section{Agradecimientos}

El apoyo financiero ha sido aportado por el Royal Norwegian Ministry of Social Welfare and Health (SHD), Collaborating Board for health promotion (SOHO), County Municipality of Sör-Tröndelarg, School Division (Trondheim), y la Norwegian University of Science and Technology (NTNU), Trondheim.

Además, el apoyo moral lo han prestado el Royal Norwegian Ministry of Education, Research, and Church Affairs, Royal Norwegian Ministry of Child and Family Affairs, Trondheim City Council, y SörTröndelag College Student Organization.

Se agradecen mucho las contribuciones al proyecto de las siguientes personas: Graham Clifford, Karl Hellemsvik, Rune Nitter, Kristian Onarheim, y Erik Sivertsen.

\section{Bibliografía}

BANDURA, A. Self-Efficacy. The Exercise of Control. (Collective School Efficacy). New York: W.H. Freeman and Company, 1997.

BATTISTA, J., ALMOND, R. The development of meaning in life. Psychiatry, 36, 409-427, 1973.

BJÖRGEN, I.A. The Truncated and the intact concept of learning. Revista Portuguesa de Educacao, 6, 23-54, 1993.

CLARKE, G., LEWINSOHN, P.M., HOPS, H. Adolescent coping with depression course. Eugene OR: Caste Publ Co, 1990.

EYSENCK, H.J., EYSENCK, S.B.G., BARRETT, P. A Revised version of the psychotism scale. Personality and Individual Differences, 6, 21-29, 1985.

JENSEN, E.N., GÖTESTAM, K.G. The teachers role in improving human relations in high school. A quasi-experimental study. The European Journal of Psychiatry, 12, 511. 1998.

KAPLAN, H.I., SADOCK, B.J. Synopsis of Psychiatry, $6^{\text {th }}$ ed. Baltimore: William \& Wilkins, 1991.
LEENARS, A.A., WENCKSTERN, S. Suicide prevention in schools. Series on health education, aging and health care. Bristol: Hemisphere Publ Co, 1991.

LINDSLEY, O.R. Why aren't effective teaching tools widely adopted? Journal of Applied Behavior Analysis, 25, 21-26, 1992.

PORTERFIELD, A.L. Does sense of humor moderate the impact of life stress on psychological and physical well-being? Journal of Research in Personality, 21, 306317, 1987.

THE ROYAL NORWEGIAN MINISTRY OF EDUCATION, RESEARCH, AND CHURCH AFFAIRS: School Reform 94. Oslo: National Centre for Educational Resources. P O Box 8194 Dep, Oslo.

THE ROYAL NORWEGIAN MINISTRY OF EDUCATION, RESEARCH, AND CHURCH AFFAIRS: Core Curriculum for Primary, Secondary and Adult Education in Norway. Oslo: National Centre for Educational Resources. P O Box 8194 Dep, Oslo.

SVEBAK, S. Revised questionnaire on the sense of humor. Scandinavian Journal of Psychology, 15, 328-331, 1974.

SVEBAK, S., MARTIN, R.A. Humor as a form of coping. In: S. Svebak \& M.J. Apter (Eds): Stress and health: A revertal theory perspective. Washington DC: Tylor \& Francis, 173-184, 1977.

SVEBAK, S. One state's agony the other's delight: Perspectives on coping and musculoskeletal complaints. In: C.D. Spielberger, J.G. Sarason, J. Strelau, J.B. Brebner (Eds): Stress and emotion (Vol 13). Washington DC: Hemisphere, pp 215-229, 1990.

SVEBAK, S. The development of the Sense of Humor Questionnaire: From SHQ to SHQ-6. International Journal of Humor Research, 9, 341-361, 1996.

URSIN, H., ENDRESEN, I.M., URSIN, G. Psychological factors and self-reports of muscle pain. European Journal of Applied Physiology, 57, 282-290, 1988.

ZUNG, W.W.K., RICHARDS, C.B., SHORT, M.J. Selfrating depression scale in an outpatient clinic. Archives of General Psychiatry, 13, 508-515, 1965.

Dirección para correspondencia:

K Gunnar Götestam

Norwegian University of Science and Technology

Department of Neuroscience

Division of Psychiatry \& Behavioural Medicine MTFS

NO-7489 Trondheim

NORWAY 